\title{
ADAM Metallopeptidase Domain 8 Measurement
}

National Cancer Institute

\section{Source}

National Cancer Institute. ADAM Metallopeptidase Domain 8 Measurement. NCI

Thesaurus. Code C147290.

The determination of the amount of ADAM metallopeptidase domain 8 present in a sample. 